\title{
Emociones en contextos académicos. Perspectivas teóricas e implicaciones para la práctica educativa en la universidad
}

\section{Paola Verónica Rita Paoloni ${ }^{1}$}

${ }^{1}$ Consejo Nacional de Investigaciones Científicas y Tecnológicas, Universidad Nacional de Río Cuarto, Río Cuarto, Córdoba

\section{República Argentina}

Correspondencia: Paola Verónica Paoloni. Azopardo № 358, C.P.: 5800. Ciudad de Río Cuarto, provincia de Córdoba. República Argentina. E-mail: paopaoloni17@hotmail.com

(C) Education \& Psychology I+D+i and Ilustre Colegio Oficial de Psicología de Andalucía Oriental 


\section{Resumen}

Aunque las emociones tienen el potencial para influir en los procesos de enseñanza y de aprendizaje, su investigación en contextos educacionales emerge lentamente y de manera fragmentada. Este artículo busca contribuir a esclarecer un campo de estudio de la Psicología Educacional que promete contribuciones interesantes para el diseño de ambientes instruccionales promotores de emociones beneficiosas para los aprendizajes. En primer lugar, presenta desarrollos que muestran la complejidad de la emoción como constructo multidimensional. En segundo lugar, identifica perspectivas tradicionales y enfoques emergentes en su estudio. En tercer lugar, desarrolla aspectos centrales de la teoría de las emociones de logro de Pekrun y colaboradores. Finalmente, propone posibles implicaciones de esta teoría para la práctica educativa en el nivel universitario y esboza horizontes viables por donde podría discurrir futura investigación al respecto.

Palabras Clave: emociones de logro, perspectivas de estudio, implicancias educativas, universidad. 


\title{
Emotions in Academic Contexts. Theoretical perspectives and implications for educational practice in college
}

\begin{abstract}
Although emotions have the potential to influence the processes of teaching and learning, its research in educational contexts emerge slowly and piecemeal. This article seeks to contribute to clarify a field of Educational Psychology that promises contributions to design instructional environments promoters of beneficial emotions to learning. First, it shows the complexity of emotion as a multidimensional construct. Second, presents traditional perspectives and emerging approaches identified in their studie. Third, developments central aspects of achievement emotions theory, understood as promising alternative that integrates emerging trends in the field. Finally, provide possible implications for educational practice and viable horizons where future research could run.
\end{abstract}

Keywords: achievement emotions, perspectives of study, educational implications, university. 


\section{Introducción}

Estudiantes y docentes significan de manera particular los contextos instruccionales en los que se desenvuelven cotidianamente. Desde el nivel inicial hasta el universitario, innumerables horas de trabajo transcurren en los salones de clases, se entretejen complejas tramas en las relaciones sociales y el logro de importantes metas -académicas, sociales, profesionales, a corto, mediano o a largo plazo- depende de la agencia individual y colectiva que se conjugan en las instituciones educativas (Paoloni, 2014). A causa de esta impronta subjetiva, los ambientes educativos están impregnados de intensas experiencias emocionales que afectan -tanto positiva como negativamente- el aprendizaje y el desempeño, promoviendo -o perjudicandoel crecimiento personal tanto de estudiantes como de docentes (Pekrun, Frenzel, Goetz y Perry, 2007). Sin embargo, sorprende que la investigación de las emociones en contextos educativos emerja tan lentamente.

Sabemos que las emociones afectan, entre otros aspectos, el ajuste académico de los alumnos, su implicación con las tareas, las estrategias autorreguladoras del aprendizaje que despliegan y las calificaciones escolares que obtienen (González-Fernández, Rinaudo y Donolo, 2010; Linnenbrink, 2007; Pekrun, 2006). No obstante y por lo general, el grueso de antecedentes con que contamos, focaliza en emociones negativas como la ansiedad o el aburrimiento. Así, Pekrun, Goetz, Titz y Perry (2002) revisaron estudios llevados a cabo entre los años 1974 y 2000 y constataron que mientras más de 1000 trabajos focalizaban en la ansiedad de los estudiantes en relación con los exámenes, no más de 9 consideraban a la esperanza y sus efectos en los aprendizajes y logros académicos obtenidos.

En definitiva, con excepción de la ansiedad ante situaciones de examen (Zeidner, 2007) o de investigaciones acerca de los antecedentes atribucionales de las emociones (Weiner, 1992, 2005), es muy poco lo que se sabe sobre el resto de las emociones que experimentan los estudiantes -y docentes-, tanto placenteras como no placenteras (Pekrun, 2005) ${ }^{1}$. Sucede que históricamente, la investigación psicológica sobre emociones focalizó su atención en

\footnotetext{
${ }^{1}$ Es preciso aclarar que en este artículo nos referimos al rol que desempeñan las emociones en los procesos de enseñanza y de aprendizaje situados en contextos académicos formales. No incluimos avances conceptuales y empíricos efectuados desde enfoques que integran a las emociones en sus planteamientos pero que no focalizan en ellas sino en las competencias, capacidades o habilidades de los sujetos para hacer un uso inteligente -o no- de las mismas. El lector interesado en diferentes enfoques sobre Inteligencia Emocional, encontrará importantes revisiones, por ejemplo, en Pena y Repetto (2008), Fernández-Berrocal y Extremera (2005), Fernandez-Berrocal
} 
las emociones negativas y en procesos maladaptativos de regulación emocional. Quizás por esto mismo, las teorías generales sobre la emoción de que disponemos actualmente, en su mayor parte fueron construidas sobre resultados relativos a emociones negativas (Cohn y Fredrickson, 2009).

Hace más de veinte años, Weiner (1992) celebraba con entusiasmo el incremento reciente en aquel entonces- de investigaciones realizadas sobre emociones en Psicología. Casi una década después, Zeidner, Boekaerts y Pintrich (2000) postulaban la necesidad de lograr avances que nos orienten respecto de cómo tratar a las emociones y a los afectos en ambientes instructivos. En escritos aún más recientes, Linnenbrink y Pekrun (2011), insisten en la necesidad de investigación que contribuya a clarificar el rol que desempeñan las emociones en las vidas académicas de los estudiantes y provean pautas fundadas acerca de cómo ayudar a estudiantes y docentes a regular sus emociones de un modo sano y productivo. Lo expuesto, sin duda, deja en claro que la investigación sobre emociones en contextos de aprendizaje se constituye en una necesidad actual dentro del campo de estudio de la Psicología Educacional.

En el marco descrito, en este artículo nos propusimos sistematizar avances conceptuales y empíricos que enfatizan la importancia de las emociones en contextos académicos. Nuestra intención es brindar aportes que, humildemente, contribuyan a esclarecer un campo de estudio que en Psicología Educacional emerge con fuerza y promete contribuciones interesantes para el diseño de ambientes instruccionales orientados a promover emociones beneficiosas para los aprendizajes. Específicamente, pretendemos: 1) mostrar la complejidad que supone considerar la emoción como constructo multidimensional; 2) identificar tendencias emergentes en su estudio; 3) presentar rasgos centrales que definen a la teoría del controlvalor de las emociones de logro, destacando vinculaciones con otros importantes constructos estudiados desde la Psicología Educacional; 4) proponer implicaciones para la práctica educativa específicamente en el nivel universitario, sugiriendo senderos por donde podría discurrir futura investigación al respecto.

\footnotetext{
y Ruiz (2008). Datos circunscriptos al ámbito universitario pueden leerse no obstante en Castejón, Cantero y
} Pérez (2008), Extremera y Fernández-Berrocal (2004), entre otros. 


\section{Emoción: definiciones, dimensiones y perspectivas teóricas}

El campo de estudio de las emociones en Psicología es una de las áreas en las que abunda una multiplicidad de modelos teóricos acompañados, paradójicamente, de un conocimiento poco preciso de su objetivo de estudio (Choliz, 2005).

Los investigadores interesados por estudiar las emociones, han ensayado diversas definiciones aunque todavía no exista una que goce de suficiente aceptación o acuerdo generalizado. Cofer y Appley, definen a la emoción como "cualquier estado mental de agitación, vehemencia o excitación de los individuos" (Cofer y Appley, 1964 en Weiner, 1992, p. 302). Para Damasio, las emociones son "un conjunto organizado de reacciones químicas y nerviosas que produce el cerebro al detectar la presencia de un estímulo emocional (...)" (Damasio, 2001 en Petri y Govern, 2006, p. 368). Otros autores, ensayan conceptualizaciones más integradoras y complejas. En tal sentido, Gross y Thompson (2009), consideran a la emoción como "una transacción entre persona y situación que empele al sujeto a prestar atención a lo que tiene un significado particular para él y que da lugar a una respuesta multisistémica, coordinada y flexible" (Gross y Thompson, 2009, p. 5). Por su parte, Chóliz (2005) la entiende como "una experiencia afectiva en cierta medida agradable o desagradable, que supone una cualidad fenomenológica característica y que compromete tres sistemas de respuesta: cognitivosubjetivo, conductual-expresivo y fisiológico-adaptativo” (Chóliz, 2005, p. 4).

Es fácil advertir la disparidad que caracteriza a las definiciones presentadas. Si no supiéramos concretamente al término al que refieren, dudo que lográsemos inferir con facilidad que se trata del mismo y, menos aun, que específicamente aluden al concepto de emoción. Petri y Govern (2006), al igual que Chóliz (2005), consideran que esta dificultad para lograr que teóricos e investigadores acuerden una definición de emoción, se debe a su naturaleza multifacética.

Las emociones son multifacéticas o multidimensionales porque en cada experiencia emocional interviene una diversidad de aspectos subjetivos (afectivos), fisiológicos, funcionales y sociales, actuando de modo coordinado. La emoción es el constructo psicológico que integra o resume, en un todo coherente, estos cuatro componentes clave de la experiencia humana (Reeve, 1994). 
La dimensión afectiva de la emoción refiere a la experiencia subjetiva que tiene sentido y razón personal (Reeve, 1994). Las emociones como estados afectivos subjetivos, hacen que experimentemos determinados sentimientos en toda su intensidad y calidad, ante un evento, situación, persona u objeto -presente, pasado o futuro; real o imaginario- (Weiner, 1992). El aspecto subjetivo de la emoción está tan estrechamente ligado a lo que entendemos por emoción que en lo cotidiano los términos 'emoción' y 'sentimiento', a menudo se utilizan como sinónimos (Gross y Thompson, 2009). Pero la emoción no solamente nos hace 'sentir' algo (amor, odio, alegría, enojo), sino que nos moviliza a actuar o a no hacerlo. Estos impulsos a actuar -o a no hacerlo-, refieren a la dimensión fisiológica de la emoción.

La dimensión fisiológica de la emoción incluye la participación de los sistemas autonómico y hormonal (Reeve, 1994). Cuando estamos emocionados nuestro cuerpo entra en un estado de activación singular -o arousal emocional- (nuestro corazón late con fuerza, la respiración se acelera, los músculos se tensan). Los impulsos a actuar ligados al surgimiento de una emoción, están asociados con cambios automáticos neuroendocrinos que anticipan respuestas comportamentales capaces de proveer apoyo metabólico a la acción para permitir su consecución (Gross y Thompson, 2009). "Estoy congelado por el miedo", "me siento atraída por usted" o "voy a explotar de alegría", son ejemplos de expresiones cotidianas que ilustran claramente esta dimensión fisiológica de la emoción. Esta activación general del organismo, nos lleva a considerar la tercera dimensión de la emoción; esto es, su componente funcional.

La dimensión funcional de la emoción se vincula a los beneficios que las emociones proporcionan en la adaptación del organismo a su entorno. Tanto a nivel cotidiano e individual como en términos evolutivos y en general, las emociones nos permiten ser más efectivos a la hora de interactuar con el entorno. En esta facilitación que las emociones propician para la adaptación del individuo a su entorno, la esfera social emerge como aspecto relevante y así, las funciones sociales de las emociones se distinguen de otras funciones netamente adaptativas (Reeve, 1994). La función social de las emociones nos lleva a considerar su cuarta dimensión constitutiva: la dimensión expresiva de las emociones.

La dimensión expresiva de las emociones refiere a su componente conductual y social. Las emociones son fenómenos sociales por naturaleza (Reeve, 1994). Mediante la postura, los gestos, las vocalizaciones y la conducta facial, las emociones son expresadas y comunicadas a 
los demás. Permanentemente emitimos mensajes emocionales a los demás e inferimos los sentimientos privados de los otros a través de sus expresiones públicas.

En definitiva, hablar de emociones no resulta sencillo porque estamos ante un constructo complejo (Gross y Thompson, 2009). Las dimensiones constituyentes de las emociones implican a la persona en su totalidad: sus sentimientos y pensamientos, su cuerpo y fisiología, sus intenciones y propósitos, su conducta (Reeve, 1994). Todos estos elementos que acompañan al proceso emocional, pueden ser identificados e incluso pueden ser estudiados por separado, pero la emoción en sí misma es una amalgama que representa el sistema actuando en su totalidad (Weiner, 1992).

\section{Enfoques tradicionales y perspectivas emergentes en el estudio de las emociones}

A través de los años, se delimitaron varias perspectivas en el estudio de las emociones. Este proceso de diferenciación en los enfoques teóricos se vio favorecido por la cualidad multidimensional del mismo objeto de estudio que cada perspectiva intentaba explicar. Siguiendo la clasificación propuesta por Reeve (1994), tradicionalmente, los distintos enfoques para el estudio de las emociones pueden agruparse en dos grandes bloques: planteamientos biológicos y planteamientos cognitivos. En términos generales podemos decir que, mientras los teóricos biologicistas entienden que las reacciones emocionales pueden ocurrir sin que intervenga la cognición, los cognitivistas por su parte, afirman que las personas no pueden experimentar una respuesta emocional sin antes evaluar cognitivamente el significado personal del estímulo (Weiner, 1992).

Aunque juntas, las perspectivas biologicistas y cognitivas, ofrecen una visión bastante comprensiva y satisfactoria del proceso de la emoción (Reeve, 1994), en la historia de la psicología de la emoción se advierte una suerte de fragmentación en propuestas que parecen esforzarse por precisar cuál de los dos aspectos -cognitivos o biológicos- ofrecen una mejor explicación de las emociones en el comportamiento humano. No obstante, esta mirada dicotómica que caracterizó el estudio de las emociones durante muchos años, parece avanzar hacia enfoques más integrales, situados y culturalistas, una tendencia que se observa también 
en el estudio de diferentes tópicos en Psicología Educacional, entre ellos, las emociones académicas (Paoloni, 2014).

Perspectivas emergentes en el estudio de las emociones. Los postulados de Gross y Thompson (2009) o la propuesta de Griffiths y Scarantino (2009), son dos ejemplos de modelos que integran desarrollos teóricos orientados hacia visiones más integrales sobre las emociones. Así, para Gross y Thompson (2009), una experiencia emocional supone una transacción constante entre persona y situación que compele a la atención por aquello que tiene un significado particular para la persona, dando lugar a respuestas multisistémicas, flexibles y coordinadas. La emoción tiene así un efecto recursivo porque origina cambios en el ambiente, lo que a su vez afecta la probabilidad del surgimiento de emociones subsecuentes y así sucesivamente. Este feedback -o intercambio constante de información- que se produce entre la respuesta emocional y los antecedentes en su surgimiento, refleja la naturaleza dinámica y recíprocamente determinada de la emoción en un contexto dado, en una corriente continua entre estímulos emocionales y respuestas comportamentales, donde lo cognitivo y lo biológico se integran en un sistema indisoluble.

Los postulados de Gross y Thompson (2009) coinciden a grandes rasgos con la perspectiva situada acerca de la emoción planteada por Griffiths y Scarantino (2009). De tal modo, estos autores insisten en que los aspectos emocionales no pueden estudiarse de forma independiente, sino que deben pensarse en relación con contextos específicos. En estos modelos socio-cognitivos y situados de la emoción, el contexto asume un papel relevante, muy diferente al desempeñado en las perspectivas biologicistas y cognitivistas acerca de la emoción.

Desde las perspectivas tradicionales, el contexto cumple un rol bastante relegado en la consideración de las emociones, quedando confinada su participación a proporcionar estímulos y a receptar las reacciones emocionales consecuentes por parte de los organismos. En tal sentido, tanto los cognitivistas como los biologicistas centran su atención en los aportes que las emociones realizan desde 'el interior' del sujeto (Griffiths y Scarantino, 2009). En cambio, las perspectivas más integrales, situadas y dinámicas de la emoción, consideran al contexto como interviniente en el proceso emocional, atendiendo en consecuencia las interrelaciones que se establecen entre aspectos personales y contextuales en la gestación, surgimiento, expresión y regulación de las emociones (Griffiths y Scarantino, 2009). Precisamente, desde 
estos enfoques integrales y situados consideramos a continuación las emociones en contextos académicos.

\section{Las emociones en contextos académicos}

El estudio de la emoción en educación no ha permanecido ajeno a los cambios más generales acontecidos en materia de investigación educacional de las últimas décadas ${ }^{2}$. La tendencia a integrar diferentes dimensiones personales en el estudio de las emociones desde enfoques contextuales o situados, puede observarse en recientes propuestas e investigaciones sobre emoción en educación (Boekaerts, 2007; Elliot y Pekrun, 2007; Ketonen y Lonka, 2012; Linnenbrink, 2007; Meyer y Turner, 2007; Pekrun et al., 2007; entre otras). Sin embargo y, como dijimos, aunque las emociones tienen el potencial de influenciar los procesos de enseñanza y de aprendizaje, su investigación en contextos educacionales emerge lentamente y de manera fragmentada (Schutz y Pekrun, 2007).

En los últimos diez años, se advierte que las líneas de trabajo entorno a las emociones en contextos académicos progresan en relativa soledad. Así, "la investigación sobre emociones en educación, y sobre emociones de logro en general, está en un estado de fragmentación. Parece que se carece de marcos integrales y esto limita el progreso teórico y empírico" (Pekrun et al., 2007, p. 14). Frente a esta situación, la teoría del control-valor de las emociones de logro de Pekrun et al. (2007), provee una perspectiva socio-cognitiva de las emociones de estudiantes y docentes, y representa una alternativa integral a la fragmentación y dispersión teórica señalada como una característica del campo de estudio de las emociones en educación en la actualidad.

La teoría del control-valor las emociones de logro de Pekrun.

Schutz y Pekrun (2007) denominan 'emociones de logro' a las emociones que están vinculadas al contexto académico y las conciben como procesos psicológicos complejos con

\footnotetext{
${ }^{2}$ Una revisión de antecedentes en investigación educacional correspondiente a la última década, permite identificar una marcada tendencia hacia perspectivas integradoras, multidimensionales, situadas y experienciales en el estudio de los procesos de aprendizaje (De Corte, 2000; Pintrich, 2000 ayb; Volet, 2001). En tal sentido, se advierte un marcado interés de los psicólogos educaciones por considerar la influencia del contexto en fenómenos psicológicos complejos, como los que tienen lugar durante los aprendizajes académicos.
} 
componentes afectivos, cognitivos, motivacionales y expresivos. Esas emociones académicas se basan, principalmente, en el modo en que profesores y alumnos perciben lo que está ocurriendo en el contexto de una actividad concreta (Meyer y Turner, 2007). Siendo más específicos, Pekrun et al. (2007) definen a las emociones de logro como aquellas directamente vinculadas con actividades y con resultados de logro.

El logro es entendido como la cualidad de actividades o de sus resultados, evaluados en relación a estándares de excelencia. De acuerdo con estas definiciones, la mayoría de las emociones que experimentan los estudiantes en contextos académicos -aunque, obviamente, no todas-, serían emociones de logro, en tanto se relacionan con comportamientos y resultados típicamente juzgados de acuerdo con estándares de calidad -tanto por los propios alumnos como por parte de los demás. Así, la rabia o la decepción que un alumno experimenta cuando se entera que no aprobó un examen valorado como importante, el orgullo que siente cuando cumple una meta de formación, la vergüenza suscitada si el profesor lo sorprendió copiando o el disfrute experimentado mientras avanza exitosamente en la resolución de una tarea que le resulta interesante, son algunos ejemplos de emociones de logro activadas en contextos académicos.

En educación, la investigación sobre las emociones de logro focalizó en general, sobre las emociones relacionadas con resultados, como la ansiedad experimentada por los alumnos ante situaciones de examen (Zeidner, 2007) o las emociones vinculadas al éxito o al fracaso escolar (Weiner, 1992, 2005). No obstante, la perspectiva asumida por Pekrun et al. (2007), implica reconocer que las emociones concernientes a actividades relacionadas con logros, también deben ser consideradas emociones de logro en sentido estricto. Así, el disfrute y el orgullo experimentados cuando se cumple una meta, la frustración o la vergüenza suscitada cuando fracasan los esfuerzos o el aburrimiento vivido en una clase cuando expone el profesor, serían ejemplos de emociones relacionadas con actividades de logro.

Pekrun et al. (2007) consideran que la teoría del control-valor de las emociones de logro es integral en un doble sentido; primero, porque integra diversidad de aspectos personales -cognitivos, metacognitivos, motivacionales, actitudinales-, vinculados con diferentes constructos estudiados en Psicología Educacional; segundo, porque integra aspectos personales y contextuales en la consideración de los procesos emocionales de estudiantes y docentes. En 
los próximos dos apartados se argumenta acerca de por qué esta teoría es integral en el doble sentido referido.

Integración de aspectos personales en la consideración de las emociones académicas.

Con la intención de proporcionar un marco integrador y de favorecer el progreso teórico y empírico respecto de las emociones académicas, Pekrun y su equipo desarrollaron la teoría del control-valor de las emociones de logro (Pekrun et al, 2002; Pekrun et al., 2007) que provee una perspectiva socio-cognitiva de las emociones de estudiantes y docentes y representa una alternativa integral a la fragmentación y dispersión teórica señalada como una característica del campo de estudio de las emociones en educación en la actualidad.

Respecto de la integración de aspectos personales en la consideración de las emociones académicas, la propuesta de estos autores integra en sus planteos, desarrollos relativos a otros importantes constructos considerados en Psicología Educacional como creencias de control, valoraciones, creencias de autoeficacia, expectativas de resultado o atribuciones de causalidad. Así, el modelo de Pekrun y colaboradores se fundamenta en evidencia empírica consistente: un vasto programa de estudios cualitativos y cuantitativos orientados a enriquecer la perspectiva teórica y metodológica acerca de los antecedentes cognitivos de las emociones de logro situadas en contextos académicos ${ }^{3}$.

La propuesta de Pekrun et al. (2007) afirma que hay dos grupos de percepciones relevantes para la activación de emociones académicas en estudiantes y en docentes: el control subjetivo de las actividades y de los resultados; y el valor subjetivo otorgado a esas actividades y resultados. Se asume que las apreciaciones que estudiantes y docentes realizan acerca del control y del valor percibido en relación con actividades académicas y con resultados ob-

\footnotetext{
${ }^{3}$ Características de los estudios cualitativos y cuantitativos que avalan esta teoría, así como los principales hallazgos obtenidos en cada uno, se recuperan y sistematizan en Pekrun et al. (2002). El considerable nivel de estudio y desarrollo que distingue a esta modelo fue plasmado en una serie de ítems que configuran un instrumento para la medición y evaluación de las emociones académicas. Conocido como AEQ (Achievement Emotions Questionnaire) de Pekrun, Goetz y Perry (2005), este instrumento logró captar a un amplio público dedicado a la educación en distintos niveles del sistema. En la actualidad la comunidad científica está haciendo un uso importante del AEQ. Se ha utilizado en investigaciones realizadas en diversos países del mundo como por ejemplo, en Bélgica (Mouratidis et. al., 2009), China y Alemania (Frenzel et al., 2007), Estados Unidos (Berg, 2008), Filipinas (Matti et al., 2009) y España (González Fernández et al., 2010).
} 
tenidos o anticipados, son antecedentes próximos en el surgimiento de las emociones académicas.

El control subjetivo de actividades de logro y de resultados, tres tipos de expectativas serían relevantes para Pekrun et al. (2007): expectativas de control de la acción, expectativas acerca de los resultados de la acción y expectativas acerca de los resultados de la situación.

Las expectativas de control de la acción refieren a las anticipaciones realizadas por el sujeto acerca de que una actividad académica podrá exitosamente ser iniciada y desempeñada (yo controlo mi actuación). De acuerdo con Pekrun et al. (2007), estas expectativas coincidirían con la propuesta de Bandura (1993) acerca de las creencias de autoeficacia, entendidas como "los juicios acerca de la capacidad para el desempeño en acciones específicas a la luz de metas específicas" (Pintrich y Schunk, 1996, p. 104).

Las expectativas acerca de los resultados de la acción por su parte, implican anticipaciones acerca de que las actividades realizadas conducirán a los resultados que uno quiere lograr (yo controlo los resultados). Como puede inferirse, tanto las expectativas de control sobre la acción como las expectativas acerca de los resultados de la acción, enfatizan la agencia de los estudiantes y el rol de sus expectativas personales como importantes mediadores cognitivos del comportamiento. Si las primeras se vinculan con creencias de autoeficacia y las segundas con las expectativas de resultados, una diferencia que podríamos señalar entre ambas expectativas es que mientras las creencias de eficacia hacen referencia a la conducta en tiempo presente, las expectativas de resultado se centran en los resultados de esa conducta proyectados en el futuro (Wigfield y Tonks, 2002).

Finalmente, las expectativas acerca de los resultados de la situación, sugieren que los resultados esperados se presentan en una situación determinada sin que medie la propia acción por parte del sujeto (los resultados dependen de la situación). Mientras las expectativas de control sobre la acción y las expectativas de resultados de la acción, se vinculan con la idea de autodeterminación, autonomía o causación personal -tan importante para la motivación intrínseca (Huertas, 1997; Pintrich y Schunk, 1996; Ryan y Deci, 2000) y para el comportamiento autorregulado (Pintrich, 2000a)-, las expectativas de resultados de la situación, se vincularían con ideas de regulación externa de la actuación, con desmotivación o con motivación extrínseca, planteadas desde la propuesta de Ryan y Deci (2000). 
Respecto al valor subjetivo atribuido a las actividades y a los resultados obtenidos, otro de los antecedentes que Pekrun et al. (2007) proponen para explicar el surgimiento de las emociones académicas-, la teoría distingue entre valores intrínsecos y valores extrínsecos.

Pekrun et al. (2007), consideran que el valor intrínseco de las actividades refiere a la valoración que alumnos realizan acerca de la actividad en sí misma, por el placer que experimentan al realizarla sin que produzca resultados relevantes para ellos. Por ejemplo, un alumno al que le gustan las Matemáticas, puede estar interesado en resolver problemas de matemáticas más allá de que esta actividad lo conduzca o no a conseguir buenas notas. Un alumno que valore intrínsecamente una actividad académica disfrutará entonces de su realización, más allá del resultado que pueda lograr, activará en consecuencia emociones positivas como entusiasmo o alegría.

Por su parte, el valor extrínseco propuesto por Pekrun et al. (2007) -como antecedente en el devenir de las emociones académicas-, refiere a la utilidad instrumental percibida por los alumnos respecto de las actividades académicas. La atención se centra así en los resultados anticipados directa o indirectamente respecto de la realización de la actividad. Por ejemplo, un estudiante que valora el estudio porque lo ayuda a obtener buenas notas (valora las buenas notas como resultado directamente asociado al estudio) o bien, porque las buenas notas contribuyen desde su perspectiva al logro de metas futuras como conseguir el trabajo que anhela (valora las buenas notas por su importancia diferida en el marco de un proyecto de vida más amplio). En el sentido de lo formulado, el valor extrínseco propuesto por Pekrun et al. (2007), se equipara a la noción de valor instrumental planteado por Eccles (2005) y al concepto de motivación instrumental desarrollado por Lens y Simons (2002).

Interacción de aspectos personales y contextuales en la consideración de las emociones académicas.

A pesar de que la teoría de las emociones de logro acentúa la importancia de aspectos cognitivos y motivacionales en el surgimiento de las emociones académicas -como las creencias de control de los aprendizajes, las expectativas de resultados o el valor atribuido a las actividades y a los resultados-, reconoce también la influencia de otros factores personales como las disposiciones genéticas y el temperamento fisiológicamente determinado- y sociales 
-como las interacciones que se originan en el aula, las características específicas de los ambientes académicos o los rasgos que definen al contexto socio-histórico más amplio.

Dentro de este marco integral en la consideración de las emociones, la teoría postula la existencia de interacciones constantes entre diferentes aspectos personales intervinientes en los procesos emocionales y entre éstos y factores del contexto. Por consiguiente, tanto los antecedentes próximos en el surgimiento de estas emociones como las manifestaciones de las emociones propiamente dichas y sus efectos, están unidos por causalidades recíprocas todo el tiempo, lo que contribuye a definir una concepción de las emociones en educación como sistemas dinámicos, donde el flujo de la interacción entre elementos personales y contextuales es constante y recíprocamente determinado. Desde este enfoque integral de la participación de aspectos personales y contextuales en el surgimiento y desarrollo de las emociones académicas, Pekrun, Cusack, Maruyama, Elliot y Thomas (2014), llevaron a cabo un estudio orientado a extender la investigación al respecto, focalizando en el feedback de logro como factor instruccional capaz de impactar positiva o negativamente en las emociones académicas experimentadas por los estudiantes.

\section{Implicaciones para la práctica educativa en el nivel universitario}

Atendiendo a la necesidad actual de diseñar intervenciones educativas que consideren los diversos desarrollos sobre emociones en general y sobre emociones académicas en particular (Goetz, Fenzel, Hall y Pekrun, 2008), en este apartado presentamos posibles implicaciones de la teoría de las emociones de logro para la práctica educativa en el nivel universitario, integrando aportes provenientes de otras áreas de estudio en Psicología Educacional.

\section{Emociones académicas y recursos cognitivos}

La teoría del control-valor de las emociones de logro asume que las emociones ayudan a focalizar la atención sobre un objeto determinado. Si un estudiante está enojado por un fracaso académico o angustiado por un examen que se avecina, probablemente experimentará dificultades para concentrarse en el estudio. Por otro lado, se asume que las emociones positivas relacionadas con una tarea académica en particular, ayudan a focalizar la atención del estudiante en las características de la actividad, beneficiando su desempeño. En tal sentido, 
Pekrun et al. (2007) encontraron que el disfrute de los estudiantes en tareas de aprendizaje, correlacionaba positivamente con su experiencia del fluir -entendida desde la perspectiva tradicional propuesta por Csikszentmihalyi (1998)- lo que permitiría suponer que emociones positivas promueven recursos cognitivos focalizados sobre el aprendizaje. Avalando estas presunciones, Pekrun et al. (2002) y Pekrun et al. (2004, en Pekrun et al., 2007) encontraron que la ansiedad, la vergüenza y la desesperanza surgidas por resultados de logro valorados como negativos, correlacionaron negativamente con la experiencia del fluir y positivamente con pensamientos irrelevantes acerca de la tarea.

En cuanto a estrategias cognitivas -otro importante recurso cognitivo-, la investigación ha puesto de manifiesto que el estado de ánimo positivo facilita maneras creativas, holísticas y más flexibles de resolver problemas, mientras que los estados afectivos negativos pueden promover una manera de pensar más rígida y analítica (Isen, 2000 en Pekrun et al., 2007). En coincidencia con estos hallazgos, emociones de activación positiva (como el disfrute, la alegría o la esperanza) favorecerían el uso de estrategias de aprendizaje más flexibles tales como la elaboración y organización de contenidos (Pekrun et al., 2002), mientras que las emociones de activación negativa (como el enojo, la frustración o la ansiedad) facilitarían el uso de estrategias más rígidas como la copia, la repetición o procedimientos simples de ensayo-error. Por su parte, las emociones de desactivación (como el aburrimiento, la tristeza o la desesperanza), irían en detrimento de cualquier tratamiento profundo de la información relacionada con la tarea (Pekrun et al., 2007).

Los postulados y hallazgos acerca de las vinculaciones entre emociones de logro y recursos cognitivos -como atención, concentración, estrategias cognitivas-, sugieren al menos dos importantes implicancias para la práctica educativa en el contexto de la universidad.

a) Relevancia de atender a las consignas que se solicitan a los estudiantes. Proponer consignas que en su formación resulten lo suficientemente claras para que promuevan en los estudiantes la elaboración de interpretaciones orientadas en el sentido de los objetivos educativos, parece ser una alternativa promisoria para el surgimiento de emociones placenteras entre los alumnos. Resultados de investigación ponen de manifiesto las complejas interacciones que se establecen entre la interpretación que los estudiantes realizan de una consigna de trabajo y la dinámica de aspectos motivacionales y estados emocionales que se sucede en consecuencia. Específicamente, en un estudio realizado por Paoloni y Rinaudo (2009) con alumnos 
universitarios avanzados, se observó que cuando los planes cognitivos inicialmente construidos por los estudiantes respecto de una consigna de trabajo que les fue solicitada, no se orientaron en el sentido esperado por los docentes, los alumnos manifestaron un elevado monto de angustia, una escasa disposición a implicarse en la tarea, poco entusiasmo y la primacía de metas dirigidas a realizar el trabajo por el sólo hecho de cumplir con un requisito solicitado (metas extrínsecas).

b) Pertinencia de contar con materiales de estudio potencialmente ricos para propiciar una intensa actividad cognitiva. Pekrun et al. (2007) aluden a la importancia que supone para la activación de estados emocionales beneficiosos para los estudiantes, el hecho de propiciar una intensa actividad cognitiva mediante, por ejemplo, el establecimiento de relaciones entre diferentes temas o problemas, disciplinas o teorías. En este sentido, la propuesta realizada por Perkins (1995), tendiente a fomentar una pedagogía para la reflexión, la comprensión y el uso inteligente del conocimiento, guardan vinculación directa con los argumentos esgrimidos por Pekrun et al. (2007). Si los alumnos perciben que las situaciones de aprendizaje los desafían a desplegar su repertorio de recursos cognitivos, si consideran que las tareas fomentan un uso inteligente del conocimiento, probablemente se predispongan a implicarse en los aprendizajes, activando en consecuencia emociones beneficiosas a tal fin. Los hallazgos obtenidos por Ainley y Ainley (2011) se orientan a fundamentar estas presunciones. Los estudios llevados a cabo por estos autores, sugieren que cuando los estudiantes perciben que los temas o contenidos propuestos en el marco de sus procesos de formación tienen relevancia personal y un significado para sus vidas, están más propensos a experimentar disfrute y entusiasmo, lo que a su vez impacta favorablemente en el compromiso desplegado para con sus aprendizajes.

\section{Emociones académicas y recursos motivacionales}

La teoría de las emociones de logro asume que las emociones de activación positiva como el disfrute, incrementan el interés y refuerzan la motivación de los estudiantes por aprender y que las emociones de desactivación negativa, como la desesperanza o el aburrimiento, conducen a un detrimento en la motivación y a un desencanto con las metas (Pekrun et al., 2007). Hallazgos de investigación llevadas a cabo en el campo de la motivación académica, se orientan en un sentido similar al propuesto por Pekrun et al. (2007). 
Investigadores como Alonso-Tapia y López-Luengo (1999), Ames (1992) y Hanrahan (1998), encontraron que la motivación intrínseca, se vincula con un incremento del interés en la tarea, mayor compromiso cognitivo, alta calidad emocional experimentada durante el proceso de aprendizaje y obtención de mejores logros académicos. Si el contexto instructivo contribuye a conformar el interés -situacional y personal- de los estudiantes e influye en los valores subyacentes a sus emociones, entonces parece importante pensar en tareas académicas que promuevan emociones positivas y el compromiso de los alumnos por implicarse en sus aprendizajes. Al respecto, podríamos sugerir las siguientes implicancias educativas.

a) Importancia de promover en los estudiantes valores intrínsecos y extrínsecos con los cuales comprometerse. Schunk y Pajares (2005) advierten que las valoraciones construidas por los estudiantes respecto de los contextos académicos son tan importantes en el devenir de sus procesos de aprendizaje que explicarían el hecho de que un alumno se comprometa en una actividad aunque sus creencias de eficacia para obtener éxito en su consecución, sean bajas. Según estos autores, explicitar a los estudiantes el valor de importancia, de interés o de utilidad que puede tener una tarea en relación con sus metas de formación profesional o proyectos de vida más amplios, es un modo de promover en ellos un mayor compromiso para con la misma y la activación de estados emocionales beneficiosos. Como se infiere, la importancia de las valoraciones para los aprendizajes académicos destacada por Schunk y Pajares (2005), se orienta exactamente en el sentido propuesto por la teoría de Pekrun acerca de los antecedentes próximos que explican el surgimiento de las emociones de logro.

b) Importancia del aprendizaje vicario y de los mecanismos de 'contagio emocional' para la activación de emociones placenteras en los estudiantes. Hallazgos de investigación sugieren que el entusiasmo de padres, docentes o tutores por la educación puede inducir en los alumnos entusiasmo por el aprendizaje y por los resultados obtenidos como parte de su proceso de formación (Pekrun et al., 2007). En igual sentido, Meyer y Turner (2007) consideran que las emociones de estudiantes y docentes están inexorablemente entrelazadas unas con otras en los salones de clase, lo que supone pensar en un co-desarrollo emocional. De acuerdo con estos planteos, cuando los docentes asumen una actitud positiva ante la tarea de enseñar, cuando se entusiasman con su rol de educadores, se 'enamoran' -o re-enamoran- de su profesión, se pondría en marcha una alternativa promisoria para entusiasmar a los estudiantes con su tarea de aprender. 
c) Necesidad de considerar la estructura de meta que predomina en las clases. La investigación sobre emociones académicas, ha constatado que la estructura de meta que predomina en las clases, ejerce efectos sobre el control subjetivo, los valores y las emociones consecuentes de los miembros que las integran (Pekrun et al., 2007). Estructuras competitivas, definidas por repetidos episodios de comparación social por ejemplo, generan emociones negativas como enojo, ansiedad o desesperanza. Por el contrario, estructuras de metas vinculadas con el dominio de la tarea propuesta, con expectativas positivas respecto de los resultados obtenidos y con parámetros intrapersonales -y no interpersonales- de valoración de logros, probablemente sean más beneficiosas en términos del control percibido por los estudiantes y de las emociones académicas que en consecuencia generan (Pekrun et al., 2007; Turner, Meyer y Schweinle, 2003).

d) Importancia de diseñar tareas académicas desafiantes en relación con las capacidades de los estudiantes. Esto favorecería el surgimiento y desarrollo de creencias de autoeficacia capaces de impactar positivamente en las valoraciones y emociones consecuentes. Ryan y Deci (2000) sostienen que si la demanda de una tarea académica es percibida por los estudiantes como demasiado difícil o demasiado fácil respecto de sus capacidades, es probable que se genere en ellos aburrimiento. Similares presunciones pueden realizarse para el caso de los docentes. Si los docentes perciben que sus estudiantes participan activamente, con preguntas desafiantes o brindando respuestas competentes, el disfrute puede verse incrementado lo que, a su vez, impactaría positivamente en el entusiasmo de sus estudiantes por aprender e implicarse en las tareas o aprendizajes propuestos (Pekrun et al., 2007).

\section{Emociones académicas y procesos de autorregulación}

Pekrun, Goetz, Frenzel, Barchfeld y Perry (2011), consideran que las emociones académicas se relacionan con los procesos de autorregulación que llevan a cabo los estudiantes, impactando en la calidad de los aprendizajes y resultados obtenidos. Así, emociones placenteras podrían promover el surgimiento de una motivación extrínseca o intrínseca, facilitar un uso más flexible de estrategias de aprendizaje y apoyar los procesos de autorregulación llevados a cabo por los estudiantes. En cambio, emociones negativas como el aburrimiento, la ira o la desesperanza, podrían reducir la motivación de los alumnos por aprender y los esfuerzos necesarios por procesar información, afectando negativamente su desempeño autorregulatorio y la posibilidad de obtener buenos resultados. 
En efecto, la investigación muestra que la oportunidad de elegir promueve en los estudiantes una forma más animada y optimista de enfrentarse a las tareas académicas (Pintrich y Schunk, 1996; Ryan y Deci, 2000). Si la valoración y las expectativas de control acerca de la tarea y de los resultados son antecedentes próximos en el surgimiento de las emociones académicas (Pekrun et al., 2007) y si los ambientes que apoyan el aprendizaje autorregulado de los estudiantes son propicios para incrementar este tipo de creencias de control y valoraciones positivas por los aprendizajes, entonces las siguientes implicancias educativas pueden resultar de interés en contextos universitarios, precisamente por la libertad y autonomía que suele caracterizarlos.

a) Importancia de ampliar el margen de autonomía con que cuentan los estudiantes en los espacios curriculares. Respecto del modo en que los docentes pueden favorecer el despliegue de mayor autonomía en sus estudiantes, Stefanou (2004, en González Fernández, 2005) propone el despliegue de tres tipos de estrategias vinculadas a diferentes ámbitos: el organizativo, el procedimental y el cognitivo. Entre las estrategias que potencian la autonomía organizativa, se proponen por ejemplo, todas aquellas orientadas a responsabilizar a los alumnos en la organización y desarrollo de alguna clase. Por su parte, entre las estrategias que favorecen el desarrollo de autonomía procedimental, el autor propone brindar a los alumnos la posibilidad de que elijan temas, materiales de estudio o el modo de demostrar el progreso logrado en sus conocimientos. Por último, entre las estrategias tendientes a desarrollar autonomía cognitiva, se sugieren las siguientes: otorgar suficiente tiempo a los alumnos en la elaboración de los trabajos requeridos, ampliando así la posiblidad de que adopten enfoques profundos de aprendizaje; generar feedback de calidad sobre el desempeño de los estudiantes para ayudarlos a identificar debilidades que pueden ser mejoradas y fortalezas que pueden ser aprovechadas; fomentar el esclarecimiento y formulación de metas personales; promover procesos de auto-evaluación, propiciar debates y alentar un pensamiento crítico. En suma, las estrategias propuestas por Stefanou (2004, en González Fernández, 2005), tienen el potencial de originar estados emocionales beneficiosos para los aprendizajes en tanto acentúan las creencias de control de los estudiantes sobre sus procesos de aprendizaje y resultados obtenidos. La universidad es precisamente un contexto propicio para considerarlas.

b) Necesidad de ayudar a los alumnos a percibir y valorar positivamente la autonomía con que cuentan para aprender. Hallazgos de investigación referidos al contexto universi- 
tario, sugieren que no basta con diseñar contextos académicos que brinden a los estudiantes espacios para que ejerzan autonomía en diferentes niveles o en relación con diversos ámbitos de actuación, más bien parece necesario orientarlos para que puedan primero reconocer esos espacios, valorarlos como importantes o instrumentales respecto de su formación profesional y progresar gradualmente hacia mayores niveles de autorregulación de los recursos internos y externos con que cuentan para aprender (Paoloni, 2010).

c) Importancia de atender al conocimiento y regulación emocional como competencias a desarrollar en los estudiantes. La teoría del control-valor propone que los educadores pueden cambiar las emociones de los estudiantes de modo indirecto, interviniendo para que modifiquen algunas de sus creencias y valoraciones acerca de aspectos personales o contextuales. En este sentido, se supone que si, por ejemplo, el re-entrenamiento atribucional ha resultado efectivo para cambiar la motivación de los estudiantes, entonces probablemente pueda ser útil para cambiar también sus emociones (Pekrun et al., 2007). Planteos similares que abogan por la posibilidad de lograr cambios en las emociones de los estudiantes a partir de una intervención inicialmente externa dentro del contexto instructivo, los encontramos en la propuesta de Boekaerts (1999) y de Boekaerts y Niemivirta (2000).

d) Importancia de considerar los beneficios del aprendizaje cooperativo para el surgimiento de emociones placenteras. González Fernández (2005) y Huertas y Montero (2001) consideran que en las tareas que propician un trabajo cooperativo tienen efectos positivos sobre la autoestima de los integrantes del grupo, sobre la percepción de control de los aprendizajes y sobre el logro académico obtenido. En igual sentido, advierten que, en general, el hecho de trabajar en torno de una tarea que promueve la cooperación, permite generar más fácilmente que otro tipo de actividades, situaciones propicias para fomentar un mayor margen de autonomía, curiosidad y nivel de dificultad óptimo, aspectos todos asociados -según la propuesta de Pekrun et al. (2007)-, con el surgimiento y desarrollo de estados emocionales beneficiosos para estudiantes y docentes. El trabajo de Rivers, Hagelskamp y Brackets (2013) se orienta en el sentido sugerido por las ideas expuestas. De acuerdo con estos autores, los grupos con positivas cualidades socio-emocionales, se caracterizan porque sus miembros se perciben unidos, valorados y apoyados por sus pares; en estos grupos priman sentimientos de confianza y respeto mutuo, vinculados a una fuerte percepción de pertenencia y al entusiasmo y disfrute como emociones consecuentes. Según los autores, la investigación ha demostrado que estas emociones y percepciones positivas de los estudiantes acerca de las relacionan interpersonales 
que se construyen en el grupo, se vinculan a su vez con un mejor desempeño académico y un mayor compromiso desplegado para con los aprendizajes (Rivers et al., 2013).

Emociones académicas y procesos de feedback.

De acuerdo con Pekrun et al. (2007), el feedback acerca del éxito o del fracaso en los aprendizajes afecta las emociones de los estudiantes. Así, generalmente, el éxito obtenido genera emociones de alegría o satisfacción, entusiasmo o esperanza; por el contrario, el fracaso suele vincularse con la experiencia de angustia, decepción, culpa o enojo. Pero además, el feedback contribuye a conformar expectativas y el valor percibido de desempeños futuros que determinan emociones prospectivas en los estudiantes. Es así como, según Pekrun et al. (2007), las emociones influyen en el aprendizaje pero también el aprendizaje y los resultados obtenidos, influyen en el surgimiento de emociones subsecuentes. Esto implica -como dijimos- que las emociones, sus efectos y sus antecedentes, están unidos por causalidades recíprocas. Los lazos que posibilitarían estas uniones entre emociones, efectos y antecedentes, tienen que ver con los ciclos de feedback que se despliegan habitualmente en un contexto académico determinado.

Pekrun et al. (2007), suponen que ciclos de feedback positivos -esto es, disfrute del aprendizaje, éxito en los exámenes y emociones positivas-, se reforzarían recíprocamente a lo largo del tiempo. No obstante, los autores consideran que los ciclos de feedback negativos no necesariamente serían perjudiciales para los aprendizajes en tanto también pueden vincularse con procesos o resultados óptimos. Por ejemplo, cuando un fracaso genera ansiedad, esta ansiedad puede inducir a una evitación exitosa del fracaso en próximas ocasiones de examen. En relación con lo expuesto podemos pensar en la siguiente implicancia para la práctica en niveles superiores de educación.

Importancia de atender al contenido del feedback, a cómo se comunica y al modo en que los estudiantes lo interpretan. Como vimos, para que sea efectivo o se asocie al surgimiento de emociones positivas en los estudiantes, un ciclo de feedback no necesariamente debe partir de comentarios positivos acerca de una actuación o resultado obtenido (Pekrun et al., 2007). Aunque sí, debe cuidar el modo en que los mensajes son comunicados y la interpretación que el alumno realiza al respecto de modo tal que su atención quede centrada en la posibilidad de mejora y en el esfuerzo personal como alternativa para lograrlo (Alexander, 
2006; Paoloni y Rinaudo, 2014). Hallazgos de investigación se orientan a avalar esta presunción, destacando la importancia que el feedback tiene para incrementar la autoeficacia percibida, cuando comunica que los errores son parte del aprendizaje, cuando vincula los progresos de los alumnos hacia la meta con los esfuerzos realizados, cuando favorece el reconocimiento del control que los estudiantes tiene sobre sus aprendizajes y cuando sugiere una visión de la habilidad como algo susceptible de ser mejorado (Pintrich y Schunk, 1996; Urdan y Turner, 2005). Si los alumnos pueden anticipar control sobre tareas o resultados subsecuentes, si además interpretan que sus capacidades o habilidades pueden mejorarse, entonces probablemente experimenten emociones prospectivas positivas como entusiasmo, disfrute o esperanza.

\section{Consideraciones para finalizar. Abriendo horizontes hacia nuevos desafíos}

En este artículo partimos de considerar la complejidad que supone el estudio de la emoción, como constructo multidimensional, para aproximarnos gradualmente a las características que definen al campo más específico de las emociones académicas. Si bien recientemente se advierte un notable incremento de investigaciones que focalizan en las emociones experimentadas por estudiantes y docentes en los salones de clase, los avances logrados derivan de enfoques diversos (miniteorías) que progresan en relativa soledad, en un estado de fragmentación que amerita esfuerzos por lograr mayores consensos entre teóricos e investigadores (Pekrun y Schutz, 2007).

¿Se supone entonces que este panorama debe resultar desalentador para quienes estamos interesados en el estudio de las emociones en educación? ¡Por supuesto que no! ¡Todo lo contrario! Las emociones académicas constituyen un campo de estudio por derecho propio, en pleno proceso de construcción, capaz de brindar contribuciones importantes que nos ayuden a entender cómo tratar a las emociones en los procesos de enseñanza-aprendizaje, cómo diseñar contextos académicos potencialmente promotores de estados y dinámicas emocionales óptimos para estudiantes y docentes.

Además de integrar tendencias actuales en investigación educacional y en el estudio de las emociones académicas en particular, la teoría de las emociones de logro de Pekrun y colaboradores, resulta especialmente interesante para ser considerada en el ámbito de la universidad. ¿Por qué decimos esto? Porque, por un lado y como vimos a lo largo de este trabajo, se trata de una teoría cuyos postulados básicos se erigen sobre dos constructos principales: las 
creencias de control de los aprendizajes y las valoraciones acerca del contexto académico. Por otro lado, porque el contexto universitario es uno de los ámbitos más indicados para promover precisamente el tipo de creencias y valoraciones que la teoría postula como antecedentes en el surgimiento y devenir emocional de estudiantes y docentes.

Cada uno de los postulados de la teoría de las emociones de logro que presentamos en este trabajo, representa una arista por donde podría discurrir futura investigación al respecto. Cada una de las posibles implicaciones educativas propuestas en este artículo, es una invitación, un desafío para espíritus inquietos preocupados por el progreso de la ciencia y la mejora de la educación, principalmente en el nivel superior. Tenemos así la semilla y un terreno fértil, tenemos entusiasmo y también algunas herramientas... imaginemos ahora lo que podemos lograr.

\section{Referencias}

Ainley, M. y Ainley, J. (2011). Student engagement with science in early adolescence: The contribution of enjoyment to students' continuing interest in learning about science. Contemporary Educational Psychology, 39, 4-12.

doi: 10.1016/j.cedpsych.2010.08.001

Alexander, P. (2006). Psychology in Learning and Instruction. Ohio: Pearson Merril Prentice Hall.

Alonso-Tapia, J. y López-Luengo, G. (1999). Efectos motivacionales de las actividades docentes en función de las motivaciones de los alumnos. En J.I. Pozo y C. Monereo (Eds.), El Aprendizaje Estratégico (pp. 35-57). Madrid: Santillana.

Ames, C. (1992). Classrooms: goals, structures, and student motivation. Journal of Education Psychology, 84(3), 261-271. doi: 10.1037/0022-0663.84.3.261

Bandura, A. (1993). Perceived self-efficacy in cognitive development and functioning. Educational Psychologist, 28(2), 117-148. Recuperado de http://jamiesmithportfolio.com/EDTE800/wp-content/PrimarySources/Bandura5.pdf

Berg, C. (2008). Academic Emotions in Student Achievement: Promoting Engagement and Critical Thinking through Lessons in Bioethical Dilemmas. Maricopa Institute for Learning. Recuperado de http://mcli.maricopa.edu/files/mil/reports/cberg-report.pdf

Boekaerts, M. (1999). Self-regulated learning: where we are today. International Journal of Educational Research, 31(6), 445-457. doi: 10.1016/S0883-0355(99)00014-2 
Boekaerts, M. (2007). Understanding Students' Affective Processes in the Classroom. En P. Schutz y R. Pekrun (Eds.), Emotion in Education (pp. 37-56). San Diego: Academic Press.

Boekaerts, M. y Niemivirta, M. (2000). Self-regulated learning. Finding a balance between learning goals and ego-protective goals. En M. Boekaerts, P. Pintrich y M. Zeidner (Eds.), Handbook of self-regulation (pp. 417-450). San Diego: Academic Press.

Castejón, J.L., Cantero, M.P. y Pérez, N. (2008). Diferencias en el perfil de competencias socio-emocionales en estudiantes universitarios de diferentes ámbitos científicos. Electronic Journal of Research in Educational Psychology, 6(2), 339-362. Recuperado de:

http://www.investigacion-psicopedagogica.org/revista/new/ContadorArticulo.php?267

Chóliz Montañés, M. (2005). Psicología de la emoción: el proceso emocional. Recuperado de www.uv.es/=choliz.

Cohn, M. y Fredrickson, B. (2011). Positive Emotions. En S. López y C.R. Snyder (Eds.), The Oxford Handbook of Positive Psychology (pp. 13-24). New York: Oxford University Press.

Csikszentmihalyi, M. (1998). Creatividad. El fluir y la psicología del descubrimiento y la invención. Barcelona: Paidós.

De Corte, E. (2000). Marrying theory building and the improvement of school practice: a permanent challenge for instructional psychology. Learning and Instruction, 10, 249266. doi: 10.1016/S0959-4752(99)00029-8

Eccles, J. (2005). Subjetive Task Value and the Model of Achievement-related Choice. En A. Elliot y C. Dweck (eds.), Handbook of Competence and Motivation (pp. 105-121). New York-London: The Guilford Press.

Elliot, A.J. y Pekrun, R. (2007). Emotion in the Hierarchical Model of Approach-Avoidance Achievement Motivation. En P. Schutz y R. Pekrun (Eds.), Emotion in Education (pp. 57-73). San Diego: Academic Press.

Extremera, N. y Fernández-Berrocal, P. (2004). El papel de la inteligencia emocional el alumnado. Evidencias empíricas. Revista Electrónica de Investigación Educativa 6(2). Recuperado de http://www.redalyc.org/articulo.oa?id=15506205

Fernández-Berrocal, P. y Extremera, N. (2005). La inteligencia emocional y la educación de las emociones. Revista Interuniversitaria de Formación del Profesorado, 19(3): 6393. Recuperado de:

http://webprueba2.quned.es/archivos_publicos/webex_actividades/4980/ieimaciel03.pdf 
Fernández -Berrocal, P. y Ruiz-Aranda, D. (2008). La inteligencia emocional en la educación. Electronic Journal of Research in Educational Psychology, 6(2), 421-436. Recuperado de:

http://www.investigacion-psicopedagogica.org/revista/new/ContadorArticulo.php?256

Frenzel, A., Thrash, T., Pekrun, R. y Goetz, T. (2007). Achievement emotions in Germany and China: A cross-cultural validation of the Academic Emotions QuestionnaireMathematics (AEQ-M). Journal of Cross-Cultural Psychology, 38 (3), 302-309. doi: $10.1177 / 0022022107300276$

Goetz, T., Frenzel, A., Hall, N. y Pekrun, R. (2008). Antecedents of academic emotions: Testing the internal/external frame of reference model for academic enjoyment. Contemporary Educational Psychology, 33, 9-33. doi: 10.1016/j.cedpsych.2006.12.002

Goleman, D. (2000). La inteligencia emocional. Por qué es más importante que el cociente intelectual. Buenos Aires: Vergara Ediciones.

González-Fernández, A. (2005). Motivación académica. Teoría, aplicación y evaluación. Madrid: Pirámide.

González- Fernández, A., Donolo, D. y Rinaudo, M.C. (2010). Motivación académica y ajuste emocional en universitarios argentinos y españoles. En P. V. Paoloni, M.C. Rinaudo, D. Donolo, A. González Fernández y N. Roselli (Comps.), Estudios sobre motivación. Enfoques, resultados y lineamientos para acciones futuras (pp. 383-403). Río Cuarto: EFUNARC.

Griffiths, P.E. y Scarantino, A. (2009). Emotions in the wild: The situated perspective on emotion. En P. Robbins y M. Aydede (Eds.), Cambridge Handbook of Situated Cognition (pp. 437-453). Cambridge: Cambridge University Press. Recuperado de http://paul.representinggenes.org/webpdfs/Griff.Scara.IP.EmotionsWild.pdf.

Hanrahan, M. (1998). The effect of learning enviroment factors on students' motivation and learning. International Journal of Science Education, 20(6), 737-753. Recuperado de http://eprints.qut.edu.au/1352/1/hanrahan_ijse.pdf

Huertas, J.A. y Montero, I. (2001). La interacción en el aula. Aprender con los demás. Buenos Aires: Aique.

Ketonen, E. y Lonka, K. (2012). Do situational academic emotions predict academic outcomes in a lecture course? Procedia - Social and Behavioral Sciences 69, 1901-1910. doi: 10.1016/j.sbspro.2012.12.144 
Lens, W. y Simons, J. (2002). From duty to desire. The role of students' future time perspective and instrumentality perceptions for study motivation and self-regulation. En F. Pajares y T. Urdan (Eds.), Academic Motivation of Adolescents (pp. 221-276). Greenwich: IAP Ediciones.

Linnenbrink, E. A. (2007). The Role of Affect in Student Learning: A Multi-Dimensional Approach to Considering the Interaction of Affect, Motivation an Engagement. En P. Schutz y R. Pekrun (Eds.), Emotion in Education (pp. 107-124). San Diego: Academic Press.

Linnenbrink-García L. y Pekrun, P. (2011). Students' emotions and academic engagement: Introduction to the special issue. Contemporary Educational Psychology, 36, 1-3. doi: 10.1016/j.cedpsych.2010.08.001

Matti, C., Tria, D. y Verano, C. (2009). Action-Control as Predictors of Learning-Related Achievement Emotions. The International Journal of Research and Review, 3, 97-119. Meyer, D.K. y Turner, J.C. (2007). Scaffolding emotions in classrooms. En P. Schutz y R. Pekrun (Eds.), Emotion in education (pp. 243-258). San Diego: Elsevier Academic Press.

Mouratidis, A., Vansteenkiste, M., Lens, W. y Vanden-Auweele, Y. (2009). Beyond positive and negative affect: Achievement goals and discrete emotions in the elementary physical education classroom. Psychology of Sport and Exercise, 10(3), 336-343. doi: 10.1016/j.psychsport.2008.11.004

Paoloni, P.V. (2010). Motivación para el aprendizaje. Aportes para su estudio en el contexto de la universidad. En P.V. Paoloni, M.C. Rinaudo, D. Donolo, A. González Fernández y N. Rosselli (Comps.), Estudios sobre motivación: enfoques, resultados, lineamientos para acciones futuras (pp. 11-370). Río Cuarto: EFUNARC.

Paoloni, P.V. (2014). El papel de las emociones en los aprendizajes académicos. En P.V. Paoloni, M.C. Rinaudo y A. González Fernández (Comps.), Cuestiones en Psicología Educacional. Perspectivas teóricas, metodológicas y estudios de campo (pp. 83-131). Tenerife: Sociedad Latina de Comunicación Social.

Paoloni, P.V. y Rinaudo, M.C. (2009). Motivación, tareas académicas y procesos de feedback. Un estudio comparativo entre alumnos universitarios. Revista Electrónica de Motivación y Emoción, 11(31). Recuperado de http://reme.uji.es/articulos/numero31/article9/texto.html.

Paoloni, P.V. y Rinaudo, M.C. (2014). Procesos de feedback desde una perspectiva multidimensional. Un estudio orientado a promover autorregulación en estudiantes 
universitarios. En P.V. Paoloni, M.C. Rinaudo y A. González Fernández (Comps.), Cuestiones en Psicología Educacional. Perspectivas teóricas, metodológicas y estudios de campo (pp. 287-323). Tenerife: Sociedad Latina de Comunicación Social.

Pekrun, R. (2005). Progress and open problems in educational emotion research. Learning and Instruction, 15, 497-506. doi: :10.1016/j.learninstruc.2005.07.014

Pekrun, R. (2006). The control-value theory of achievement emotions: Assumptions, corollaries, and implications for educational research and practice. Educational Psychology Review, 18, 315-341. doi: 10.1007/s10648-006-9029-9

Pekrun, R. Cusack, A. Maruyama, K. Elliot, A. y Thomas, K. (2014). The power of anticipated feedback: Effects on students' achievement goals and achievement emotions. Learning and Instruction, 29, 115-124. doi: 10.1016/j.learninstruc.2013.09.002

Pekrun, R. y Schutz, P. (2007). Where Do We Go from Here? Implications and Future Directios for Inquiry on Emotions in Education. En P. Schutz y R. Pekrun (Eds.), Emotion in Education (pp. 313-331). California: Academic Press.

Pekrun, R., Frenzel, A., Goetz, T. y Perry, R. (2007). The Control-Value Theory of Achievement Emotions: An Integrative Approach to Emotions in Education. En P. Schutz y R. Pekrun (Eds.), Emotion in Education (pp. 13-36). California: Academic Press.

Pekrun, R., Goetz, T. y Perry, R. (2005) Achievement Emotions Questionnaire. User's Manual. Munich: University of Munich. Recuperado de http://es.scribd.com/doc/217451779/2005-AEQ-Manual

Pekrun, R., Goetz, T., Frenzel, A., Barchfeld, P. y Perry, R. (2011). Measuring emotions in students` learning and performance: The Achievement Emotions Questionnaire (AEQ). Contemporary Educational Psychology, 36, 36-48. doi: 10.1016/j.cedpsych.2010.10.002

Pekrun, R., Goetz, T., Titz, W. y Perry, R. (2002). Academic emotions in students' selfregulated learning and achievement: a program of qualitative and quantitative research. Educational Psychologist, 37(2), 91-106. doi: 10.1207/S15326985EP3702_4

Pena-Garrido, M. y Repetto-Talavera, E. (2008). Estado de investigación en España sobre Inteligencia Emocional en el ámbito educativo. Electronic Journal of Research in Educational Psychology, 6(2), 400-420. Recuperado de http://www.investigacionpsicopedagogica.org/revista/new/ContadorArticulo.php?244

Perkins, D. (1995). La escuela inteligente. Del adiestramiento de la memoria a la educación de la mente. Barcelona: Gedisa. 
Petri, H. y Govern, J. (2006). Motivación. Teoría, investigación y aplicaciones. México: Thomson Learning.

Pintrich, P. (2000a). The role of goal orientation in self-regulated learning. En M. Boekaerts, P. Pintrich y M. Zeidner (Eds.), Handbook of self-regulation (pp. 451-502). San Diego: Academic Press.

Pintrich, P. (2000b). Educational Psychology at the Millennium: A Look Back and a Look Forward. Educational Psychologist, 35(4), 221-231. doi: 10.1207/S15326985EP3504_01

Pintrich, P. y Schunk, D. (1996). Motivation in Education: theory, research and applications. New Jersey: Prentice Hall.

Reeve, J. (1994). Motivación y Emoción. Madrid: Mc Graw Hill.

Rivers, S.E., Hagelskamp, C. y Brackett, M.A. (2013). Understanding and assessing the social-emotional attributes of classrooms. En J. McMillan (Ed.), Sage Handbook of Research on Classroom Assessments (pp. 347-366). Thousand Oaks: Sage Publications.

Ryan, R. y Deci, E. (2000). Intrinsic and Extrinsic Motivations: Classic Definitions and New Directions. Contemporary Educational Psychology, 25, 54-67. doi: 10.1006/ceps.1999.1020

Schunk, D. y Pajares, F. (2005). Competence Perceptions and Academic Functioning. En A. Elliot y C. Dweck (Eds.), Handbook of Competence and Motivation (pp. 85-104). New York-London: The Guilford Press.

Schutz, P. y Pekrun, R. (2007). Emotion in Education. San Diego: Academic Press.

Turner, J., Meyer, D. y Schweinle, A. (2003). The importance of emotion in theories of motivation: empirical, methodological, and theoretical considerations from a goal theory perspective. Educational Research, 39, 375-393. doi: 10.1016/j.ijer.2004.06.005

Urdan, T. y Turner, J. (2005). Competence Motivation in the Classroom. En A. Elliot y C. Dweck (Eds.), Handbook of Competence and Motivation (pp. 297-317). New York: The Guilford Press.

Volet, S. (2001). Understanding Learning and Motivation in Context: A multi-dimensional and multi-level cognitive-situative perspective. En S. Volet y S. Järvelä (Eds.), Motivation in Learning Contexts. Theoretical Advances and Methodological Implications, (pp. 57-82). Londres: Pergamon-Elsevier.

Weiner, B. (1992). Human Motivation. Metaphors, Theories and Research. London: SAGE Publications. 
Weiner, B. (2005). Motivation from an Attribution Perspective and the Social Psychology of Perceived Competence. En A. Elliot y C. Dweck (Eds.), Handbook of Competence and Motivation (pp. 73-84). New York-London: The Guilford Press.

Wigfield, A. y Tonks, S. (2002). Adolescents' expectancies for success and achievement task values during the middle and high school years. En F. Pajares y T. Urdan (Eds.), Academic Motivation of Adolescents (pp. 53-82). Greenwich: IAP Ediciones.

Zeidner, M. (2007). Test Anxiety in Educational Contexts: Concepts, Findings, and Future Directios. En P. Schutz y R. Pekrun (Eds.), Emotion in Education (pp. 165-184). San Diego: Academic Press.

Zeidner, M., Boekaerts, M. y Pintrich, P. (2000). Self-regulation. Directions and Challenges for Future Research. En M. Boerkaerts, P. Pintrich y M. Zeidner (Eds.), Handbook of Self-regulation (pp. 749-768). San Diego: Academic Press. 